\title{
A nonlinear Discrete Duality Finite Volume Scheme for convection-diffusion equations
}

\author{
Clément Cancès, Claire Chainais-Hillairet and Stella Krell
}

\begin{abstract}
We introduce a nonlinear DDFV scheme for a convection-diffusion equation. The scheme conserves the mass, satisfies an energy-dissipation inequality and provides positive approximate solutions even on very general grids. Numerical experiments illustrate these properties.
\end{abstract}

Key words: Discrete Duality Finite Volume scheme, nonlinear stability, discrete entropy/dissipation relation

MSC (2010): 65M08, 35B40.

\section{Motivation}

Many parabolic problems arising for instance in porous media flows or semiconductor devices modelling have an underlying variational structure and can be interpreted as gradient flows in appropriate geometries. When an energy-dissipation relation is established, it gives some information on the long-time asymptotics of the problem. When designing numerical schemes for such kind of problems, it is interesting to mimic the energy-dissipation relation at the discrete level.

In this work, we focus on the most basic drift-diffusion equation. Let $\Omega$ be a polygonal connected open bounded subset of $\mathbb{R}^{2}$ and let $T>0$ be a finite time

Clément Cancès

Inria Lille - Nord Europe, Team RAPSODI, 40 avenue Halley, 59650 Villeneuve d'Ascq, France

e-mail: clement.cances@inria.fr

Claire Chainais-Hillairet

Univ. Lille, CNRS,UMR 8524-Laboratoire Paul Painlevé. F-59000 Lille, France

e-mail: Claire.Chainais@math.univ-lille1.fr

Stella Krell

Université Côte d'Azur, CNRS, Inria, LJAD, France

e-mail: stella.krell@unice.fr 
horizon. The problem writes:

$$
\begin{aligned}
& \partial_{t} u+\operatorname{div} \mathbf{J}=0, \mathbf{J}=-\nabla u-u \nabla V, \text { in } \Omega \times(0, T) \\
& \mathbf{J} \cdot \mathbf{n}=0, \text { on } \partial \Omega \times(0, T) \\
& u(\cdot, 0)=u_{0} \text { in } \Omega,
\end{aligned}
$$

with the following assumptions on the data:

(A1) The initial data $u_{0}: \Omega \rightarrow \mathbb{R}_{+}$is measurable and such that

$$
\int_{\Omega} u_{0} d x>0 \text { and } \int_{\Omega} H\left(u_{0}\right) d x<\infty,
$$

where $H(s)=s \log s-s+1$ for all $s \geq 0$.

(A2) The exterior potential $V$ belongs to $C^{1}(\bar{\Omega}, \mathbb{R})$.

The free energy $\mathbb{E}$ and the dissipation $\mathbb{I}$ for (1) are defined respectively by

$$
\mathbb{E}(t)=\int_{\Omega}(H(u)+V u)(x, t) d x, \quad \mathbb{I}(t)=\int_{\Omega} u|\nabla(\log u+V)|^{2} d x .
$$

They are linked by the relation $\frac{d \mathbb{E}}{d t}+\mathbb{I}=0$. As $u$ is nonnegative, $\mathbb{I}$ is a dissipation and the energy is decaying with time. Moreover, when time goes to infinity, the solution $u$ to (1) converges towards the steady-state $u_{\infty}=\left(\int_{\Omega} u_{0} d x / \int_{\Omega} e^{-V} d x\right) e^{-V}$.

On admissible meshes, it is possible to write a classical two-point finite volume scheme in order to approximate (1). With the Scharfetter-Gummel numerical fluxes, the preservation of the steady-state is ensured, as a discrete counterpart of the entropy-dissipation property (see [4]). In this paper, we want to design a scheme which satisfies the same properties but that could be applicable on almost general meshes including non-conformal and distorted meshes. We propose a Discrete Duality Finite Volume scheme. Let us mention that a robust free energy diminishing finite volume scheme based on the VAG scheme has already been proposed and analyzed in [3].

\section{Presentation of the numerical scheme}

\subsection{Meshes and notations}

In order to define a DDFV scheme, as for instance in [6, 2], we need to introduce three different meshes - the primal mesh, the dual mesh and the diamond mesh and some associated notations.

The primal mesh denoted $\overline{\mathfrak{M}}$ is composed of the interior primal mesh $\mathfrak{M}$ (a partition of $\Omega$ with polygonal control volumes) and the set $\partial \mathfrak{M}$ of boundary edges seen 
as degenerate control volumes. For all $K \in \overline{\mathfrak{M}}$, we define $x_{K}$ the center of $K$. The family of centers is denoted by $\mathfrak{X}=\left\{x_{K}, K \in \overline{\mathfrak{M}}\right\}$.

Let $\mathfrak{X}^{*}$ denote the set of the vertices of the primal control volumes in $\overline{\mathfrak{M}}$. Distinguishing the interior vertices from the vertices lying on the boundary, we split $\mathfrak{X}^{*}$ into $\mathfrak{X}^{*}=\mathfrak{X}_{i n t}^{*} \cup \mathfrak{X}_{\text {ext }}^{*}$. To any point $x_{K^{*}} \in \mathfrak{X}_{\text {int }}^{*}$, we associate the polygon $K^{*}$, whose vertices are $\left\{x_{K} \in \mathfrak{X} / x_{K^{*}} \in \bar{K}, K \in \mathfrak{M}\right\}$. The set of these polygons defines the interior dual mesh denoted by $\mathfrak{M}^{*}$. To any point $x_{K^{*}} \in \mathfrak{X}_{\text {ext }}^{*}$, we then associate the polygon $K^{*}$, whose vertices are $\left\{x_{K^{*}}\right\} \cup\left\{x_{K} \in X / x_{K^{*}} \in \bar{K}, K \in \overline{\mathfrak{M}}\right\}$. The set of these polygons is denoted by $\partial \mathfrak{M}^{*}$ called the boundary dual mesh and the dual mesh is $\mathfrak{M}^{*} \cup \partial \mathfrak{M}^{*}$, denoted by $\overline{\mathfrak{M}^{*}}$.

For all neighboring primal cells $K$ and $L$, we assume that $\partial K \cap \partial L$ is a segment, corresponding to an edge of the mesh $\mathfrak{M}$, denoted by $\sigma=K \mid L$. Let $\mathscr{E}$ be the set of such edges. We similarly define the set $\mathscr{E}^{*}$ of the edges of the dual mesh. For each couple $\left(\sigma, \sigma^{*}\right) \in \mathscr{E} \times \mathscr{E}^{*}$ such that $\sigma=K \mid L=\left(x_{K^{*}}, x_{L^{*}}\right)$ and $\sigma^{*}=K^{*} \mid L^{*}=\left(x_{K}, x_{L}\right)$, we define the quadrilateral diamond cell $\mathscr{D}_{\sigma, \sigma^{*}}$ whose diagonals are $\sigma$ and $\sigma^{*}$, as shown on Figure 1. If $\sigma \in \mathscr{E} \cap \partial \Omega$, we note that the diamond degenerates into a triangle. The set of the diamond cells defines the diamond mesh $\mathfrak{D}$. It is a partition of $\Omega$.

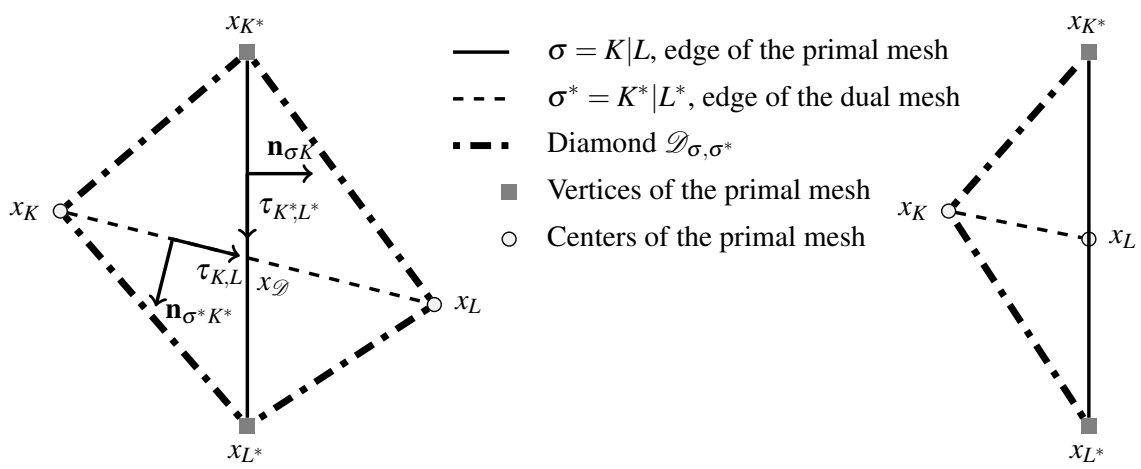

Fig. 1 Definition of the diamonds $\mathscr{D}_{\sigma, \sigma^{*}}$ and related notations.

Finally, the DDFV mesh is made of $\mathscr{T}=\left(\overline{\mathfrak{M}}, \overline{\mathfrak{M}^{*}}\right)$ and $\mathfrak{D}$. For each primal or dual cell $M\left(M \in \overline{\mathfrak{M}}\right.$ or $\left.M \in \overline{\mathfrak{M}^{*}}\right)$, we define $\mathrm{m}_{M}$ the measure of $M, \mathscr{E}_{M}$ the set of the edges of $M$ (it coincides with the edge $\sigma=M$ if $M \in \partial \mathfrak{M}$ ), $\mathfrak{D}_{M}$ the set of diamonds $\mathscr{D}_{\sigma, \sigma^{*}} \in \mathfrak{D}$ such that $\mathrm{m}\left(\mathscr{D}_{\sigma, \sigma^{*}} \cap M\right)>0$, and $d_{M}$ the diameter of $M$.

For a diamond $\mathscr{D}_{\sigma, \sigma^{*}}$, whose vertices are $\left(x_{K}, x_{K^{*}}, x_{L}, x_{L^{*}}\right)$, we define: $x_{\mathscr{D}}$ the center of the diamond cell $\mathscr{D}:\left\{x_{\mathscr{D}}\right\}=\sigma \cap \sigma^{*}, \mathrm{~m}_{\sigma}$ the length of the primal edge $\sigma$, $\mathrm{m}_{\sigma^{*}}$ the length of the dual edge $\sigma^{*}, \mathrm{~m}_{\mathscr{D}}$ the measure of $\mathscr{D}, d_{\mathscr{D}}$ its diameter, $\theta_{\mathscr{D}}$ the angle between $\left(x_{K}, x_{L}\right)$ and $\left(x_{K^{*}}, x_{L^{*}}\right)$. We will also use two direct basis $\left(\tau_{K^{*} L^{*}}, \mathbf{n}_{\sigma K}\right)$ and $\left(\mathbf{n}_{\sigma^{*} K^{*}}, \tau_{K, L}\right)$, where $\mathbf{n}_{\sigma K}$ is the unit normal to $\sigma$ outward $K, \mathbf{n}_{\sigma^{*} K^{*}}$ is the unit normal to $\sigma^{*}$ outward $K^{*}, \tau_{K^{*}, L^{*}}$ is the unit tangent vector to $\sigma$, oriented from $K^{*}$ to $L^{*}, \tau_{K, L}$ is the unit tangent vector to $\sigma^{*}$, oriented from $K$ to $L$. 


\subsection{Discrete unknowns and discrete operators}

We need several types of degrees of freedom to represent scalar and vector fields in the discrete setting. Let us introduce $\mathbb{R}^{\mathscr{T}}$ the linear space of scalar fields constant on the cells of $\overline{\mathfrak{M}}$ and $\overline{\mathfrak{M}^{*}}$ :

$$
u_{\mathscr{T}} \in \mathbb{R}^{\mathscr{T}} \Longleftrightarrow u_{\mathscr{T}}=\left(\left(u_{K}\right)_{K \in \overline{\mathfrak{M}}},\left(u_{K^{*}}\right)_{K^{*} \in \overline{\mathfrak{M}^{*}}}\right)
$$

and $\left(\mathbb{R}^{2}\right)^{\mathfrak{D}}$ the linear space of vector fields constant on the diamonds, $\xi_{\mathfrak{D}}=$ $\left(\xi_{\mathscr{D}}\right)_{\mathscr{D} \in \mathfrak{D}}$. We define the scalar products $\llbracket \cdot, \cdot \rrbracket \mathscr{T}$ on $\mathbb{R}^{\mathscr{T}}$ and $(\cdot, \cdot)_{\mathfrak{D}}$ on $\left(\mathbb{R}^{2}\right)^{\mathfrak{D}}$ by

$$
\begin{aligned}
\llbracket v_{\mathscr{T}}, u_{\mathscr{T}} \rrbracket_{\mathscr{T}} & =\frac{1}{2}\left(\sum_{K \in \mathfrak{M}} \mathrm{m}_{K} u_{K} v_{K}+\sum_{K^{*} \in \overline{\mathfrak{M}^{*}}} \mathrm{~m}_{K^{*}} u_{K^{*}} v_{K^{*}}\right), \forall u_{\mathscr{T}}, v_{\mathscr{T}} \in \mathbb{R}^{\mathscr{T}}, \\
\left(\xi_{\mathfrak{D}}, \varphi_{\mathfrak{D}}\right)_{\mathfrak{D}} & =\sum_{\mathscr{D} \in \mathfrak{D}} \mathrm{m}_{\mathscr{D}} \xi_{\mathscr{D}} \cdot \varphi_{\mathscr{D}}, \quad \forall \xi_{\mathfrak{D}}, \varphi_{\mathfrak{D}} \in\left(\mathbb{R}^{2}\right)^{\mathfrak{D}} .
\end{aligned}
$$

The DDFV method is based on the definitions of a discrete gradient, of a discrete divergence and a duality formula.The discrete gradient has been introduced in [5] and developed in [6]. The discrete divergence has been introduced in [6]. We recall here the definition of the discrete gradient. The discrete gradient is a mapping from $\mathbb{R}^{\mathscr{T}}$ to $\left(\mathbb{R}^{2}\right)^{\mathfrak{D}}$ defined for all $u_{\mathscr{T}} \in \mathbb{R}^{\mathscr{T}}$ by $\nabla^{\mathfrak{D}} u_{\mathscr{T}}=\left(\nabla^{\mathscr{D}} u_{\mathscr{T}}\right)_{\mathscr{D} \in \mathfrak{D}}$, where

$$
\nabla^{\mathscr{D}} u_{\mathscr{T}}=\frac{1}{\sin \left(\boldsymbol{\theta}_{\mathscr{D}}\right)}\left(\frac{u_{L}-u_{K}}{\mathrm{~m}_{\sigma^{*}}} \mathbf{n}_{\sigma K}+\frac{u_{L^{*}}-u_{K^{*}}}{\mathrm{~m}_{\sigma}} \mathbf{n}_{\sigma^{*} K^{*}}\right), \quad \forall \mathscr{D} \in \mathfrak{D} .
$$

For $u_{\mathscr{T}} \in \mathbb{R}^{\mathscr{T}}$ and $\mathscr{D} \in \mathfrak{D}$, we define $\delta^{\mathscr{D}} u_{\mathscr{T}}=\left(\begin{array}{c}u_{K}-u_{L} \\ u_{K^{*}}-u_{L^{*}}\end{array}\right)$. Then, for all $u_{\mathscr{T}}, v_{\mathscr{T}} \in \mathbb{R}^{\mathscr{T}}$, we can write

$$
\left(\nabla^{\mathfrak{D}} u_{\mathscr{T}}, \nabla^{\mathfrak{D}} v_{\mathscr{T}}\right)_{\mathfrak{D}}=\sum_{\mathscr{D} \in \mathfrak{D}} \delta^{\mathscr{D}} u_{\mathscr{T}} \cdot \mathbb{A}^{\mathscr{D}} \delta^{\mathscr{D}} v_{\mathscr{T}}
$$

where

$$
\mathbb{A}^{\mathscr{D}}=\frac{1}{4 \mathrm{~m}_{\mathscr{D}}}\left(\begin{array}{cc}
\mathrm{m}_{\sigma}^{2} & \mathrm{~m}_{\sigma} \mathrm{m}_{\sigma^{*}} \mathbf{n}_{\sigma K} \cdot \mathbf{n}_{\sigma^{*} K^{*}} \\
\mathrm{~m}_{\sigma} \mathrm{m}_{\sigma^{*}} \mathbf{n}_{\sigma K} \cdot \mathbf{n}_{\sigma^{*} K^{*}} & \mathrm{~m}_{\sigma^{*}}^{2}
\end{array}\right) \quad \forall \mathscr{D} \in \mathfrak{D} .
$$

Finally, we introduce a reconstruction operator on diamonds $r^{\mathfrak{D}}$. It is a mapping from $\mathbb{R}^{\mathscr{T}}$ to $\mathbb{R}^{\mathfrak{D}}$ defined for all $u_{\mathscr{T}} \in \mathbb{R}^{\mathscr{T}}$ by $r^{\mathfrak{D}} u_{\mathscr{T}}=\left(r^{\mathscr{D}} u_{\mathscr{T}}\right)_{\mathscr{D} \in \mathfrak{D}}$, where for $\mathscr{D} \in$ $\mathfrak{D}$, whose vertices are $x_{K}, x_{L}, x_{K^{*}}, x_{L^{*}}$,

$$
r^{\mathscr{D}} u_{\mathscr{T}}=\frac{1}{4}\left(u_{K}+u_{L}+u_{K^{*}}+u_{L^{*}}\right) .
$$




\subsection{The scheme}

Let $\Delta t$ be a time step. We first discretize the initial condition by taking the mean values of $u_{0}$ and the exterior potential $V$ by taking its nodal values on the primal and dual cells. It defines $u_{\mathscr{T}}^{0}$ and $V_{\mathscr{T}}$. Then, for all $n \geq 0$, we look for $u_{\mathscr{T}}^{n+1} \in\left(\mathbb{R}_{+}^{*}\right)^{\mathscr{T}}$ solution to:

$$
\begin{gathered}
\left\|\frac{u_{\mathscr{T}}^{n+1}-u_{\mathscr{T}}^{n}}{\Delta t}, \psi_{\mathscr{T}}\right\|_{\mathscr{T}}+T_{\mathfrak{D}}\left(u_{\mathscr{T}}^{n+1} ; g_{\mathscr{T}}^{n+1}, \psi_{\mathscr{T}}\right)=0, \quad \forall \psi_{\mathscr{T}} \in \mathbb{R}^{\mathscr{T}}, \\
T_{\mathfrak{D}}\left(u_{\mathscr{T}}^{n+1} ; g_{\mathscr{T}}^{n+1}, \psi_{\mathscr{T}}\right)=\sum_{\mathscr{D} \in \mathfrak{D}} r^{\mathscr{D}} u_{\mathscr{T}}^{n+1} \delta^{\mathscr{D}} g_{\mathscr{T}}^{n+1} \cdot \mathbb{A}^{\mathscr{D}} \delta^{\mathscr{D}} \psi_{\mathscr{T}}, \\
g_{\mathscr{T}}^{n+1}=\log \left(u_{\mathscr{T}}^{n+1}\right)+V_{\mathscr{T}} .
\end{gathered}
$$

The scheme is written here under a compact form. But it can also be expanded on primal and dual meshes after the introduction of conservative numerical fluxes.

\section{Some mathematical properties of the scheme}

The goal of this section is to highlight some important properties of the numerical scheme. If a solution to (2) exists then it satisfies the mass conservation, the control of energy and dissipation and it is positive. This allows to show the existence of a discrete solution to the scheme. The proofs will be detailed in a forthcoming long article in which the convergence of the scheme will also be established. In what follows, $\left(u_{\mathscr{T}}^{n}\right)_{n \geq 1} \in\left(\mathbb{R}_{+}^{*}\right)^{\mathscr{T} \times \mathbb{N}^{*}}$ denotes a solution to the scheme (2).

We first establish the conservation of the mass at the discrete level. It is obtained by induction and by choosing $\psi_{\mathscr{T}}=\left((1)_{K \in \overline{\mathfrak{M}}},(0)_{K^{*} \in \overline{\mathfrak{M}}^{*}}\right)$ and $\left((0)_{K \in \overline{\mathfrak{M}}},(1)_{K^{*} \in \overline{\mathfrak{M}}^{*}}\right)$ in $(2 a)$.

Lemma 1. If $\left(u_{\mathscr{T}}^{n}\right)_{n \geq 0}$ is a solution to the scheme (2), it satisfies

$$
\sum_{K \in \overline{\mathfrak{M}}} u_{K}^{n} m_{K}=\sum_{K^{*} \in \overline{\mathfrak{M}}^{*}} u_{K^{*}}^{n} m_{K^{*}}=\int_{\Omega} u_{0}(x) d x, \forall n \geq 0 .
$$

Let us now establish the discrete counterpart of the energy-dissipation property. Therefore, we define the discrete energy and the discrete dissipation:

$$
\mathbb{E}_{\mathscr{T}}^{n}=\llbracket H\left(u_{\mathscr{T}}^{n}\right)+V_{\mathscr{T}} u_{\mathscr{T}}^{n}, 1_{\mathscr{T}} \rrbracket_{\mathscr{T}}, \quad \mathbb{I}_{\mathfrak{D}}^{n}=T_{\mathfrak{D}}\left(u_{\mathscr{T}}^{n} ; g_{\mathscr{T}}^{n}, g_{\mathscr{T}}^{n}\right)
$$

The following result is obtained by choosing $\psi_{\mathscr{T}}=g_{\mathscr{T}}^{n+1}$ in $(2 \mathrm{a})$ and by using the convexity of $H$.

Lemma 2. If $\left(u_{\mathscr{T}}^{n}\right)_{n \geq 0}$ is a solution to the scheme (2), it satisfies

$$
\frac{\mathbb{E}_{\mathscr{T}}^{n+1}-\mathbb{E}_{\mathscr{T}}^{n}}{\Delta t}+\mathbb{I}_{\mathfrak{D}}^{n+1} \leq 0
$$


The relation (4) directly implies that the sequence $\left(\mathbb{E}_{\mathscr{T}}^{n}\right)_{n>0}$ is non-increasing since the dissipation is non-negative. We can adapt the path proposed in [3, §3] to our context and prove that the solutions to the scheme remain bounded away from 0 .

Lemma 3. Let $\left(u_{\mathscr{T}}^{n}\right)_{n \geq 0}$ be a solution to the scheme (2). There exists $\boldsymbol{\varepsilon}_{\mathscr{T}, \Delta t}>0$ depending on $\mathscr{T}, \Delta t, \Omega$ and $V$ such that for all $0 \leq n \leq N$

$$
u_{K}^{n+1} \geq \varepsilon_{\mathscr{T}, \Delta t}, \quad \forall K \in \overline{\mathfrak{M}} \quad \text { and } \quad u_{K^{*}}^{n+1} \geq \varepsilon_{\mathscr{T}, \Delta t}, \quad \forall K^{*} \in \overline{\mathfrak{M}^{*}} .
$$

The lower bound $\varepsilon_{\mathscr{T}, \Delta t}$ depends on the mesh and on the time step. But for a fixed grid and a fixed time step $\Delta t$, the estimate (5) (together with (4)) ensures that the solution remains in a compact subdomain of $\left(\mathbb{R}_{+}^{*}\right)^{\mathscr{T} \times \mathbb{N}^{*}}$ on which the scheme is uniformly continuous. Thanks to a topological degree argument, this allows to prove the existence of (at least) one solution to the scheme. The proof mimics the one of [3, Proposition 3.8].

Proposition 1. Let $u_{0}$ and $V$ satisfying assumptions (A1) and (A2), let $\mathscr{T}$ be a $D D F V$ mesh of $\Omega$ as in $\S 2.1$, then there exists (at least) one solution $\left(u_{\mathscr{T}}^{n}\right)_{n \geq 0} \in$ $\left(\mathbb{R}_{+}^{*}\right)^{\mathscr{T} \times \mathbb{N}^{*}}$ to the scheme (2).

\section{Numerical experiments}

We test our method on a test case proposed in [3]. We set $\Omega=(0,1)^{2}$, and $V\left(x_{1}, x_{2}\right)=-x_{1}$. The exact solution $u_{\mathrm{ex}}$ is then defined by

$$
u_{\mathrm{ex}}\left(\left(x_{1}, x_{2}\right), t\right)=e^{-\alpha t+\frac{x_{1}}{2}}\left(\pi \cos \left(\pi x_{1}\right)+\frac{1}{2} \sin \left(\pi x_{1}\right)\right)+\pi e^{\left(x_{1}-\frac{1}{2}\right)}
$$

with $\alpha=\pi^{2}+\frac{1}{4}$. We choose $u_{0}=u_{\mathrm{ex}}(\cdot, 0)$. Note that $u_{0}$ vanishes on $\left\{x_{1}=1\right\}$.

In order to illustrate the convergence and the robustness of our method, we test its convergence on two sequences of meshes. The first sequence of primal meshes is made of successively refined Kershaw meshes. The second sequence of primal meshes is the so-called quadrangle meshes mesh_quad_i of the FVCA8 benchmark on incompressible flows. One mesh of each sequence is depicted in Figure 2. In the refinement procedure, the time step is divided by 4 when the mesh size is divided by 2 .

The nonlinear system (2) is solved thanks to Newton's method. In order to avoid the singularity of the log near 0 , the sequence $\left(u_{\mathscr{T}}^{n+1, i}\right)_{i>0}$ to compute $u_{\mathscr{T}}^{n+1}$ from the previous state $\left(u_{\mathscr{T}}^{n}\right)_{i>0}$ is initialized by $u_{\mathscr{T}}^{n+1,0}=\max \left(u_{\mathscr{T}}^{n}, 10^{-12}\right)$. As a stopping criterion, we require the $\ell^{1}$-norm of the residual to be smaller than $10^{-10}$. In Tables 1 and 2 , the quantities erru and errgu respectively denote the $L^{\infty}\left((0, T) ; L^{2}(\Omega)\right)$ error on the solution and the $L^{2}(\Omega \times(0, T))^{2}$ error on the gradient, whereas ordu and or- 
dgu are the corresponding convergence orders. It appears that the method is slightly more than second order accurate w.r.t. space.

The maximal (resp. mean) number of Newton iterations by time step is denoted by $N_{\max }$ (resp. $N_{\text {mean }}$ ). We observe that the needed number of Newton iterations starts from a reasonably small value and falls down to 1 after a small number of time steps. Therefore, our method does not imply an important extra computational cost when compared to linear methods. Eventually, we can check that the minimal value $\min u_{\mathscr{T}}^{n}$ remains strictly greater than 0 , as proved in Lemma 3 .

\begin{tabular}{|c|c||c|c|c|c|c|c|c|}
\hline $\mathrm{M}$ & $\mathrm{dt}$ & errgu & ordgu & erru & ordu & $N_{\max }$ & $N_{\text {mean }}$ & Min $u^{n}$ \\
\hline 1 & $2.0 \mathrm{E}-03$ & $2.280 \mathrm{E}-02$ & - & $3.591 \mathrm{E}-03$ & - & 9 & 2.06 & $9.996 \mathrm{E}-02$ \\
\hline 2 & $5.0 \mathrm{E}-04$ & $7.088 \mathrm{E}-03$ & 1.72 & $8.979 \mathrm{E}-04$ & 2.04 & 8 & 1.4 & $2.564 \mathrm{E}-02$ \\
\hline 3 & $1.25 \mathrm{E}-04$ & $3.512 \mathrm{E}-03$ & 1.75 & $2.670 \mathrm{E}-04$ & 3.02 & 7 & 1.07 & $6.454 \mathrm{E}-03$ \\
\hline 4 & $3.125 \mathrm{E}-05$ & $2.205 \mathrm{E}-03$ & 1.63 & $9.960 \mathrm{E}-05$ & 3.45 & 7 & 1.02 & $1.652 \mathrm{E}-03$ \\
\hline 5 & $1.563 \mathrm{E}-05$ & $1.562 \mathrm{E}-03$ & 1.55 & $5.846 \mathrm{E}-05$ & 2.40 & 6 & 1.01 & $8.396 \mathrm{E}-04$ \\
\hline
\end{tabular}

Table 1 Numerical results on the Kershaw mesh family, final time $\mathrm{T}=0.25$.

\begin{tabular}{|c|c||c|c|c|c|c|c|c|}
\hline $\mathrm{M}$ & $\mathrm{dt}$ & errgu & ordgu & erru & ordu & $N_{\max }$ & $N_{\text {mean }}$ & Min $u^{n}$ \\
\hline 1 & $4.032 \mathrm{E}-03$ & $1.696 \mathrm{E}-01$ & - & $2.061 \mathrm{E}-02$ & - & 9 & 2.24 & $1.903 \mathrm{E}-01$ \\
\hline 2 & $1.008 \mathrm{E}-03$ & $6.283 \mathrm{E}-02$ & 1.43 & $5.124 \mathrm{E}-03$ & 2.00 & 9 & 2.05 & $5.259 \mathrm{E}-02$ \\
\hline 3 & $2.520 \mathrm{E}-04$ & $2.246 \mathrm{E}-02$ & 1.56 & $1.300 \mathrm{E}-03$ & 2.08 & 8 & 1.98 & $1.287 \mathrm{E}-02$ \\
\hline 4 & $6.300 \mathrm{E}-05$ & $8.862 \mathrm{E}-03$ & 1.41 & $3.281 \mathrm{E}-04$ & 2.08 & 7 & 1.26 & $3.388 \mathrm{E}-03$ \\
\hline 5 & $1.250 \mathrm{E}-05$ & $2.733 \mathrm{E}-03$ & 1.399 & $7.284 \mathrm{E}-05$ & 1.78 & 7 & 1.01 & $7.068 \mathrm{E}-04$ \\
\hline
\end{tabular}

Table 2 Numerical results on the Quadrangle mesh family, final time $\mathrm{T}=0.25$.

The discrete stationary solution $u_{\mathscr{T}}^{\infty}$ is defined by $u_{K}^{\infty}=\rho e^{-V\left(x_{K}\right)}$ and $u_{K^{*}}^{\infty}=$ $\rho^{*} e^{-V\left(x_{K^{*}}\right)}$ for $K \in \overline{\mathfrak{M}}$ and $K^{*} \in \overline{\mathfrak{M}}^{*}$, the quantities $\rho$ and $\rho^{*}$ being fixed so that $\sum_{K \in \overline{\mathfrak{M}}} u_{K}^{\infty} m_{K}=\sum_{K \in \overline{\mathfrak{M}}^{*}} u_{K^{*}}^{\infty} m_{K^{*}}=\int_{\Omega} u_{0}(x) d x$. In order to give an evidence of the good large-time behavior of our scheme, we plot in Figure 2 the evolution of the relative energy

$$
\mathbb{E}_{\mathscr{T}}^{n}-\mathbb{E}_{\mathscr{T}}^{\infty}=\llbracket u_{\mathscr{T}}^{n} \log \left(\frac{u_{\mathscr{T}}^{n}}{u_{\mathscr{T}}^{\infty}}\right)-u_{\mathscr{T}}^{n}+u_{\mathscr{T}}^{\infty}, 1_{\mathscr{T}} \rrbracket_{\mathscr{T}}, \quad n \geq 0
$$

computed on the Kershaw meshes. We observe the exponential decay of the relative energy, recovering on general grids the behavior of the Scharfetter-Gummel scheme [4]. Similar results are observed with the quadrangle mesh sequence. 

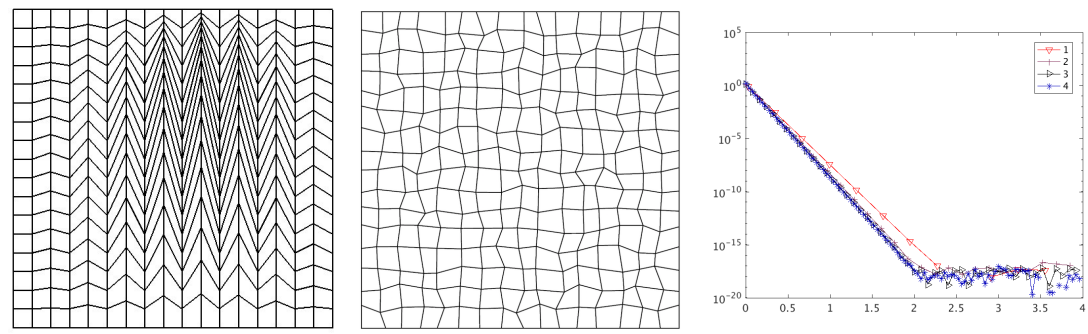

Fig. 2 Left: First Kershaw mesh. Middle: Third quadrangle mesh. Right: Discrete relative energy $\mathbb{E}_{\mathscr{T}}^{n}-\mathbb{E}_{\mathscr{T}}^{\infty}$ as a function of $n \Delta t$ computed on the first four Kershaw meshes.

\section{Conclusion}

We proposed a nonlinear DDFV scheme for drift diffusion equations that preserves the positivity of the solutions and enforces the decay of the free energy. These properties can be theoretically proved. Moreover, it appears in the numerical experiments that the scheme is second order accurate w.r.t. space, and that it captures in an accurate way the long-time asymptotic of the solution. Therefore, the scheme offers an alternative on general grids to the Scharfetter-Gummel scheme (that requires meshes with an orthogonality condition). The proof of the convergence of the numerical method will be developed in a forthcoming contribution. Note that for this purpose, it is necessary to add a nonlinear stabilization term inspired from [1].

Acknowledgements The authors are supported by the Inria teams RAPSODI and COFFEE, the LabEx CEMPI (ANR-11-LABX-0007-01) and the GEOPOR project (ANR-13-JS01-0007-01).

\section{References}

1. Andreianov, B., Bendahmane, M., Karlsen, K.H.: Discrete duality finite volume schemes for doubly nonlinear degenerate hyperbolic-parabolic equations. J. Hyperbolic Differ. Equ. 7(1), $1-67(2010)$

2. Andreianov, B., Boyer, F., Hubert, F.: Discrete duality finite volume schemes for Leray-Lionstype elliptic problems on general 2D meshes. Numer. Methods Partial Differential Equations 23(1), 145-195 (2007)

3. Cancès, C., Guichard, C.: Numerical analysis of a robust free energy diminishing finite volume scheme for parabolic equations with gradient structure. Found. Comput. Math. (2016). URL http://dx.doi.org/10.1007/s10208-016-9328-6

4. Chatard, M.: Asymptotic Behavior of the Scharfetter-Gummel Scheme for the Drift-Diffusion Model. In: FVCA VI. Springer Berlin Heidelberg (2011)

5. Coudière, Y., Vila, J.P., Villedieu, P.: Convergence rate of a finite volume scheme for a twodimensional convection-diffusion problem. M2AN Math. Model. Numer. Anal. 33(3), 493-516 (1999)

6. Domelevo, K., Omnes, P.: A finite volume method for the Laplace equation on almost arbitrary two-dimensional grids. M2AN Math. Model. Numer. Anal. 39(6), 1203-1249 (2005) 